3. P. R. Griffiths and J. A. de Haseth, Fourier Transform Infrared Spectrometry, Chemical Analysis, Vol. 83, P. J. Elving and J. D. Winefordner, Eds. (John Wiley \& Sons, New York, 1986).

4. Robert T. Conley, Infrared Spectroscopy (Allyn and Bacon, Boston, 1972), 2nd ed.

5. V. F. Kalasinsky, S. Pechsiri, and K. S. Kalasinsky, J. Chrom. Sci. 24, 543 (1986).

6. W. M. Coleman III and Bert M. Gordon, Appl. Spectrosc. 43, 305 (1989).

7. Mark M. Rochkind, Anal. Chem. 40, 762 (1968).
8. Chemical and Biological Studies of New Cigarette Prototypes That Heat Instead of Burn Tobacco (R. J. Reynolds Tobacco Co., Winston-Salem, North Carolina, 1988).

9. R. L. Stedman, Chem. Rev. 68, 153 (1968).

10. C. J. Pouchert, The Aldrich Library of FT-IR Spectra (Aldrich Chemical Co., Milwaukee, Wisconsin, 1985), 1st ed., Vols. 1 and 2.

11. Sadtler Standard Infrared Grating Spectra Library (Sadtler Research Laboratories, Philadelphia, Pennsylvania, 1985).

\title{
Wavelength Calibration of Optical Multichannel Detectors in Combination with Single- and Double-Grating Monochromators
}

\author{
MICHAEL LINDRUM and BERNHARD NICKEL* \\ Max-Planck-Institut für Biophysikalische Chemie, Abteilung Spektroskopie, Am Fassberg, D-3400 Göttingen, Federal Republic \\ of Germany
}

\begin{abstract}
For the measurement of a spectrum with an optical multichannel detector (OMCD), the relation $N=N(\lambda)$ or $\lambda=\lambda(N)$ between the channel number $N$ and the wavelength $\lambda$ must be known. For an ideal OMCD, $N(\lambda)$ and $\lambda(N)$ are completely defined by the physical parameters of the monochromator and the channel spacing of the OMCD. Series expansions $N$ $=N_{0}+\Sigma_{k} A_{k}\left(\lambda-\lambda_{0}\right)^{k}$ and $\lambda=\lambda_{0}+\Sigma_{k} B_{k}\left(N-N_{0}\right)^{k}$ are given for singlegrating monochromators $(k=1,2,3)$ and double-grating monochromators $(k=1,2)$. The validity of the series expansions has been checked experimentally and numerically for a double monochromator.

Index Headings: Emission spectroscopy; Hollow cathode lamps; Instrumentation, optical multichannel analyzer; UV-visible spectroscopy.
\end{abstract}

\section{INTRODUCTION}

For the measurement of a spectrum with a monochromator and an optical multichannel detector (OMCD), the relation between the wavelength $\lambda$ and the channel number $N$ must be known. The linear dispersion of a single-grating monochromator (SGM) or a double-grating monochromator (DGM) is nearly constant within the detection range of a small $O M C D$, and the relation between $\lambda$ and $N$ of an ideal OMCD can always be accurately enough represented by truncated series expansions

$$
\begin{gathered}
N \approx N^{*}=N_{0}+\sum_{k=1}^{n} A_{k}{ }^{j}\left(\lambda_{0}\right)\left(\lambda-\lambda_{0}\right)^{k} \\
\lambda \approx \lambda^{*}=\lambda_{0}+\sum_{k=1}^{n} B_{k}{ }^{j}\left(\lambda_{0}\right)\left(N-N_{0}\right)^{k}
\end{gathered}
$$

where $\lambda_{0}$ and $N_{0}$ are the center wavelength and the center channel number of the OMCD, and the superscript $j$

\footnotetext{
Received 24 April 1989.

* Author to whom correspondence should be sent.
}

specifies the type of monochromator $(j=s: \mathrm{SGM}, j=d$ : DGM). One way of using Eq. 1 or 2 that always works is to consider the coefficients $A_{k}{ }^{j}$ and $B_{k}{ }^{j}$ as purely empirical instrument-specific quantities. ${ }^{1}$ This empirical procedure has two major disadvantages: First, for every new value of $\lambda_{0}$ a new set of parameter values $A_{k}{ }^{j}$ and $B_{k}{ }^{j}$ must be determined. Second, it is difficult to distinguish an optical distortion of a spectrum by the OMCD from the inconstancy of the dispersion of the monochromator within the spectral range of the OMCD. Both disadvantages are avoided if, as far as possible, the known physical parameters of the monochromator are used for the determination of the $A_{k}{ }^{j}$ and $B_{k}{ }^{j}$. In this paper we derive quantitative expressions for the coefficients $A_{k}{ }^{s}, B_{k}{ }^{s}(k=$ $1,2,3)$ of an SGM and $A_{k}{ }^{d}, B_{k}{ }^{d}(k=1,2)$ of a DGM, and we apply these expressions to a DGM in combination with a particular type of OMCD.

\section{RELATION BETWEEN WAVELENGTH AND CHANNEL NUMBER OF AN IDEAL OMCD}

A monochromator with plane grating(s) and with an Ebert or Czerny-Turner mount can be characterized by the following physical parameters (see also Fig. 1):

$d$ : period (groove spacing) of the grating;

$m$ : diffraction order;

2є: Ebert angle (constant angle between the directions of incident beam and diffracted beam);

$\psi_{0}$ : rotational angle of the grating at the monochromator wavelength $\lambda_{0}$ (relative to $\bar{\psi}_{0}=0^{\circ} \dagger$ in zeroth order);

$f:$ focal length.

We start from the general grating equation ${ }^{2-4}$ for rays in planes perpendicular to the groove direction of the grating:

† Angles $\bar{\mu}$ measured in degrees are distinguished by a bar from angles $\mu$ measured in radians: $\bar{\mu}=\left(360^{\circ} / 2 \pi\right) \mu$. 


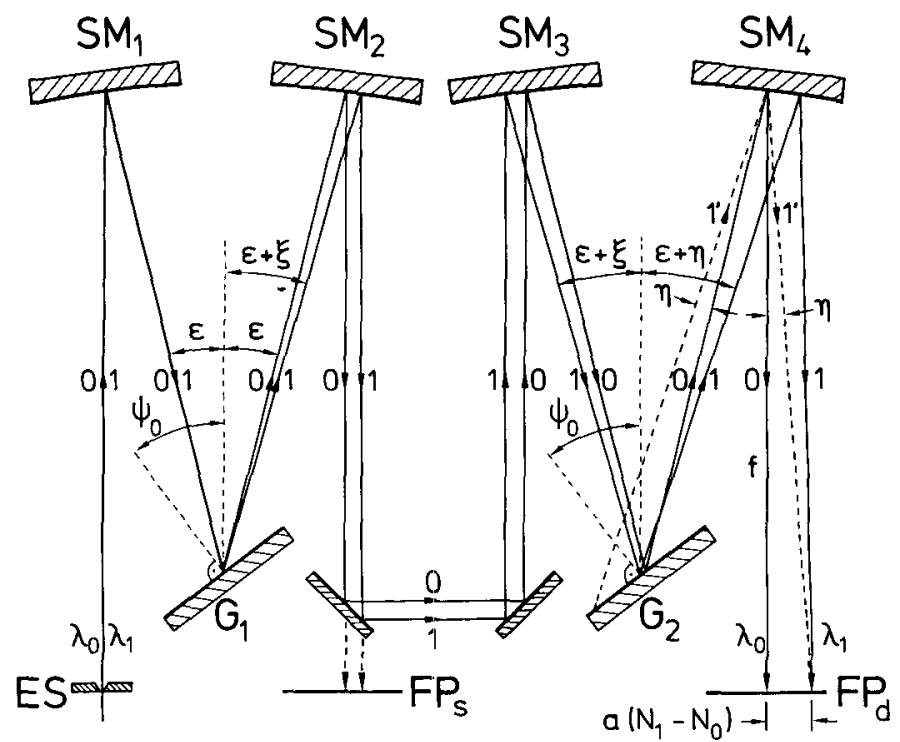

Fig. 1. Main rays 0 and 1 in a Czerny-Turner DGM for two wavelengths $\lambda_{0}$ and $\lambda_{1}>\lambda_{0}$. The dashed auxiliary ray $1^{\prime}$ has been drawn to illustrate the relation $\tan \eta=a\left(N_{1}-N_{0}\right) / f$. Abbreviations: ES: entrance slit; $\mathrm{FP}_{\mathrm{s}}$ and $\mathrm{FP}_{\mathrm{d}}$ : exit focal planes of SGM and DGM; G: grating; SM: spherical mirror.

$$
(m / d) \lambda=\sin \alpha+\sin \beta,
$$

where the angles $\alpha$ of the incident beam and $\beta$ of the diffracted beam refer to the grating normal. We simplify the notation by defining the quantity

$$
\Lambda=(m / d) \lambda \text {. }
$$

Then for an SGM and for a DGM with two equal stages (see Fig. 1) the relation

$$
\Lambda_{0}=\sin \alpha_{0}+\sin \beta_{0}=2 \sin \psi_{0} \cos \epsilon
$$

holds, since for a monochromator

$$
\begin{aligned}
& \alpha_{0}=\psi_{0}-\epsilon, \\
& \beta_{0}=\psi_{0}+\epsilon .
\end{aligned}
$$

Single-Grating Monochromator as Polychromator. When the monochromator is used as a polychromator in combination with an OMCD, then, in an SGM or in the first stage of a DGM and for wavelengths $\lambda \neq \lambda_{0}$, Eq. 5 is to be replaced by

$$
\Lambda_{0}+\Delta \Lambda=\sin \alpha_{0}+\sin \left(\beta_{0}+\xi\right) .
$$

An approximate expression for $\xi$ as a function of $\Delta \Lambda$ is obtained in three steps: (1) From Eq. 8 the exact expression for $\sin \xi$ is derived; (2) $\sin \xi$ is expanded into a power series $g(\Delta \Lambda)$ with terms up to the order of $(\Delta \Lambda)^{3} ;(3) \xi$ is obtained from $g(\Delta \Lambda)$ by using the series expansion $\xi=$ $\arcsin g=g+\frac{1}{6} g^{3}+\ldots$ and by again retaining only terms of the order of $(\Delta \Lambda)^{3}$ :

$$
\begin{aligned}
\xi \approx & \frac{1}{\cos \left(\psi_{0}+\epsilon\right)} \Delta \Lambda+\frac{\sin \left(\psi_{0}+\epsilon\right)}{2 \cos ^{3}\left(\psi_{0}+\epsilon\right)} \cdot(\Delta \Lambda)^{2} \\
& +\frac{1+2 \sin ^{2}\left(\psi_{0}+\epsilon\right)}{6 \cos ^{5}\left(\psi_{0}+\epsilon\right)}(\Delta \Lambda)^{3}
\end{aligned}
$$

In order to obtain from Eq. 9 the relation between the channel number $N$ and $\Delta \Lambda$, we assume, first, that the OMCD has a constant channel spacing $a$ and is free of optical or electrostatic distortions, and, second, that the focal plane is perpendicular to the main ray (ray 0 in Fig. 1) of the diffracted beam. With these assumptions, the relation between $\Delta N=N-N_{0}$ and $\xi$ is (see Fig. 1, where the analogous relation for $\eta$ is illustrated):

$$
a \Delta N / f=\tan \xi \approx \xi+\frac{1}{3} \xi^{3} .
$$

Insertion of $\xi$ from Eq. 9 into Eq. 10 and retaining only terms up to order $(\Delta \Lambda)^{3}$ yields

$$
\begin{aligned}
\tan \xi \approx & \frac{1}{\cos \left(\psi_{0}+\epsilon\right)} \Delta \Lambda+\frac{\sin \left(\psi_{0}+\epsilon\right)}{2 \cos ^{3}\left(\psi_{0}+\epsilon\right)} \cdot(\Delta \Lambda)^{2} \\
& +\frac{1}{2 \cos ^{5}\left(\psi_{0}+\epsilon\right)}(\Delta \Lambda)^{3} .
\end{aligned}
$$

Finally, by combination of Eqs. 1, 4, 10, and 11 the coefficients $A_{k}^{s}$ are obtained:

$$
\begin{gathered}
A_{1}{ }^{s}=\frac{1}{\cos \left(\psi_{0}+\epsilon\right)} \times \frac{f}{a} \times \frac{m}{d}, \\
A_{2}{ }^{s}=\frac{\sin \left(\psi_{0}+\epsilon\right)}{2 \cos ^{3}\left(\psi_{0}+\epsilon\right)} \times \frac{f}{a} \times\left(\frac{m}{d}\right)^{2}, \\
A_{3}{ }^{s}=\frac{1}{2 \cos ^{5}\left(\psi_{0}+\epsilon\right)} \times \frac{f}{a} \times\left(\frac{m}{d}\right)^{3} .
\end{gathered}
$$

$A_{1}^{s} \cdot a$ is the linear dispersion of an $\mathrm{SGM}^{2-4}$ [in contrast to the angular dispersion $\left.A_{1}{ }^{s} \cdot(a / f)\right]$.

An approximate expression of $\Delta \Lambda$ as a function of $\xi$ is derived by series expansion of $\sin \left(\beta_{0}+\xi\right)$ in Eq. 8 and by taking Eqs. 5 to 7 into account:

$$
\begin{aligned}
\Delta \Lambda \approx \cos \left(\psi_{0}+\epsilon\right) \xi-\frac{1}{2} & \sin \left(\psi_{0}+\epsilon\right) \xi^{2} \\
& -\frac{1}{6} \cos \left(\psi_{0}+\epsilon\right) \xi^{3}
\end{aligned}
$$

Insertion of $\xi=\arctan (a \Delta N / f) \approx(a \Delta N / f)-\frac{1}{3}(a \Delta N / f)^{3}$

into Eq. 13 yields a relation between $\Delta \Lambda$ and $\Delta N$, from which-with Eq. 4 and by comparison with Eq. 2-the coefficients $B_{k}^{s}$ of Eq. 2 are obtained:

$$
\begin{gathered}
B_{1}^{s}=\cos \left(\psi_{0}+\epsilon\right) \times \frac{d}{m} \times \frac{a}{f}, \\
B_{2}{ }^{s}=-\frac{1}{2} \sin \left(\psi_{0}+\epsilon\right) \times \frac{d}{m} \times\left(\frac{a}{f}\right)^{2}, \\
B_{3}{ }^{s}=-\frac{1}{2} \cos \left(\psi_{0}+\epsilon\right) \times \frac{d}{m} \times\left(\frac{a}{f}\right)^{3} .
\end{gathered}
$$


Double-Grating Monochromator as Polychromator. In the second stage of a DGM, Eq. 5 is to be replaced by

$$
\Lambda_{0}+\Delta \Lambda=\sin \left(\alpha_{0}-\xi\right)+\sin \left(\beta_{0}+\eta\right) .
$$

We restrict the derivation of expansion coefficients ${A_{k}}^{d}$ and $B_{k}{ }^{d}$ to $k \leq 2$, since the use of the very complex expressions $A_{3}{ }^{\mathrm{d}}=A_{3}{ }^{d}\left(\psi_{0}, \epsilon\right)$ and $B_{3}{ }^{d}=B_{3}{ }^{d}\left(\psi_{0}, \epsilon\right)$ would offer no computational advantage over the exact numerical calculation of $N=N(\lambda)$ (see below). An approximate relation between $\Delta \Lambda$ and $\eta$ is obtained by series expansion of the two terms on the right side of Eq. 15, by insertion of $\xi$ from Eq. 9, by omitting terms higher than quadratic in $\Delta \Lambda$ and $\eta$, and by using Eqs. 6 and 7:

$$
\begin{aligned}
& 2 \sin \psi_{0} \cos \psi_{0}(\Delta \Lambda)^{2}+4 \cos ^{2}\left(\psi_{0}+\epsilon\right) \cos \psi_{0} \cos \epsilon \Delta \Lambda \\
& \quad+\sin \left(\psi_{0}+\epsilon\right) \cos ^{3}\left(\psi_{0}+\epsilon\right) \eta^{2}-2 \cos ^{4}\left(\psi_{0}+\epsilon\right) \eta \approx 0
\end{aligned}
$$

Both approximate solutions of Eq. 16 are needed: $\eta$ as a function of $\Delta \Lambda$ and $\Delta \Lambda$ as a function of $\eta$ :

$$
\begin{gathered}
\eta \approx \frac{2 \cos \psi_{0} \cos \epsilon}{\cos ^{2}\left(\psi_{0}+\epsilon\right)} \Delta \Lambda+\frac{\cos ^{2} \psi_{0}}{\cos ^{4}\left(\psi_{0}+\epsilon\right)} \\
\cdot\left[2 \cos ^{2} \epsilon \tan \left(\psi_{0}+\epsilon\right)+\tan \psi_{0}\right](\Delta \Lambda)^{2}, \\
\Delta \Lambda \approx \frac{\cos ^{2}\left(\psi_{0}+\epsilon\right)}{2 \cos \psi_{0} \cos \epsilon} \eta-\frac{\cos ^{2}\left(\psi_{0}+\epsilon\right)}{8 \cos \psi_{0} \cos ^{3} \epsilon} \\
\cdot\left[2 \cos ^{2} \epsilon \tan \left(\psi_{0}+\epsilon\right)+\tan \psi_{0}\right] \eta^{2} .
\end{gathered}
$$

Finally, the relation between $\eta$ and $a, \Delta N, f$ is required. Because of the neglect of the higher-than-quadratic terms of $\eta$, the following simple relation can be used:

$$
\eta \approx \tan \eta=a \Delta N / f \text {. }
$$

By using Eqs. 4 and 19 and by comparing Eqs. 17 and 18 with Eqs. 1 and 2, we obtain the coefficients $A_{k}{ }^{d}$ and $B_{k}^{d}$ for a DGM:

$$
\begin{aligned}
A_{1}{ }^{d} & =\frac{2 \cos \psi_{0} \cos \epsilon}{\cos ^{2}\left(\psi_{0}+\epsilon\right)} \times \frac{f}{a} \times \frac{m}{d}, \\
A_{2}{ }^{d}= & \frac{\cos ^{2} \psi_{0}}{\cos ^{4}\left(\psi_{0}+\epsilon\right)} \\
& \cdot\left[2 \cos ^{2} \epsilon \tan \left(\psi_{0}+\epsilon\right)+\tan \psi_{0}\right] \\
& \times \frac{f}{a} \times\left(\frac{m}{d}\right)^{2}, \\
B_{1}{ }^{d}= & \frac{\cos ^{2}\left(\psi_{0}+\epsilon\right)}{2 \cos _{0} \cos \epsilon} \times \frac{d}{m} \times \frac{a}{f}, \\
B_{2}{ }^{d}= & -\frac{\cos ^{2}\left(\psi_{0}+\epsilon\right)}{8 \cos _{0} \cos ^{3} \epsilon} \\
& \cdot\left[2 \cos ^{2} \epsilon \tan \left(\psi_{0}+\epsilon\right)+\tan \psi_{0}\right] \\
& \times \frac{d}{m} \times\left(\frac{a}{f}\right)^{2} .
\end{aligned}
$$

$A_{1}{ }^{d} \cdot a$ is the linear dispersion of the DGM. The com-

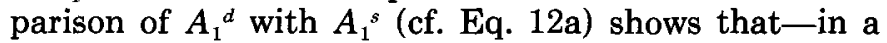
DGM with two equal stages and with $\bar{\epsilon}>0^{\circ}$-the linear dispersion is more than twice that of an SGM.

\section{EXPERIMENTAL VERIFICATION}

The validity of Eqs. 20 and 21 was checked experimentally. The monochromator was a home-made DGM with an off-plane Ebert mountł and with additive dispersion. The following physical parameters of the DGM were known:

$$
\begin{gathered}
f \approx 600 \mathrm{~mm}, \quad \bar{\epsilon} \approx 2.48^{\circ}, \\
1 / d=3600 \text { grooves } / \mathrm{mm}, \quad m=1 .
\end{gathered}
$$

The OMCD was a proximity-focused MCP-intensified detector with 1024 channels and a channel spacing of $a$ $=0.025 \mathrm{~mm}$ (Spectroscopy Instruments, Model IRY-1024/ S). An iron-neon hollow-cathode lamp (Hamamatsu, Type No. L233-26NU) was used as spectral light source. The following procedure was applied:

1. In four different spectral ranges with $\lambda_{0} \approx 250,300$, 350 , and $400 \mathrm{~nm}$, spectral lines $\lambda_{i}$ were measured with the OMCD. Peak channel numbers $N_{i}$ were determined with an accuracy of \pm 0.2 channel. Literature values $\lambda_{i}$ of wavelengths were taken from Ref. 5 . In Tables I and II, the measured spectral lines and channel numbers are listed for the two extreme spectral ranges.

2. A fixed center channel $N_{0}=500$ and a preliminary constant value $\bar{\epsilon}=2.48^{\circ}$ were chosen.

3. For each spectral range, the center wavelength $\lambda_{0}=$ $\lambda_{0}\left(N_{0}\right)$ and $f$ were determined by a least-squares fitting procedure with the condition

$$
\Sigma_{i}\left(N_{i}-N_{i}^{*}\right)^{2}=F\left(\lambda_{0}, f ; \epsilon, d, a\right)=\text { minimum }
$$

where the $N_{i}{ }^{*}$ are the channel numbers calculated with Eq. 1 and the coefficients (20).

4. The optimal value of $f$ slightly decreased with increasing wavelength from $f=604.69 \pm 0.13 \mathrm{~mm}$ at $\lambda_{0} \approx$ $250 \mathrm{~nm}$ to $f=603.74 \pm 0.24 \mathrm{~mm}$ at $\lambda_{0} \approx 400 \mathrm{~nm}$. This small but significant dependence of $f$ on $\lambda_{0}$ indicated a small error in the value of $\bar{\epsilon}$. Hence $\bar{\epsilon}$ was also treated as an adjustable parameter. The optimized parameter values for the whole spectral range from $\lambda_{0}=250 \mathrm{~nm}$ to $\lambda_{0}=400 \mathrm{~nm}$ were:

$$
\bar{\epsilon}=2.404^{\circ} \pm 0.013^{\circ}, \quad f=605.47 \pm 0.12 \mathrm{~mm} \text {. }
$$

Once accurate values of $f$ and $\bar{\epsilon}$ are known, $\lambda_{0}$ is the only free parameter. An approximate value of $\lambda_{0}$ is known from the monochromator wavelength setting. For the determination of an accurate value of $\lambda_{0}$, in principle the channel number $N_{i}$ of only one known spectral line $\lambda_{i}$ is

¥ In an off-plane Ebert or Czerny-Turner SGM with spherical mirrors, the spectral resolution is in general bad because of considerable coma. In an off-plane DGM of the same type, however, the coma cancels almost completely. 
TABLE I. Literature wavelength values $\lambda_{i}$ of spectral lines and measured peak channel numbers $N_{i}$. The other channel numbers were calculated with the common parameters $N_{0}=500, \lambda_{0}=249.8973 \mathrm{~nm}$, and $\bar{\psi}_{0}=26.75724^{\circ}: N^{*}$ with Eq. 1 and the coefficients (20), $N^{*}$ with Eqs. 22-25, and $N^{\prime}$ with Eq. 2 and the coefficients (21) and with the condition $\lambda_{i}{ }^{*}\left(N_{i}^{\prime}\right)=\lambda_{i}$. The differences $\lambda_{i}^{*}-\lambda_{i}$ with $\lambda_{i}^{*}=\lambda_{i}^{*}\left(N_{i}\right)$ from Eq. 2 illustrate the high accuracy of the measurement of wavelengths, which is roughly 10 times higher than the spectral resolution.

\begin{tabular}{cccccc}
\hline$\lambda_{i} / \mathrm{nm}$ & $N_{i}$ & $N^{*}$ & $N^{e}$ & $N^{\prime}$ & $\left(\lambda^{*}-\lambda_{i}\right) / \mathrm{nm}$ \\
\hline 247.97761 & 110.8 & 110.94 & 110.87 & 110.91 & 0.00051 \\
248.32713 & 181.3 & 181.40 & 181.36 & 181.38 & 0.00043 \\
248.81426 & 280.0 & 279.87 & 279.86 & 279.87 & -0.00064 \\
249.06441 & 331.0 & 330.57 & 330.56 & 330.57 & -0.00209 \\
250.11323 & 544.0 & 544.08 & 544.08 & 544.08 & 0.00043 \\
251.08348 & 743.3 & 742.96 & 742.98 & 742.97 & -0.00162 \\
252.28494 & 991.0 & 991.06 & 991.21 & 991.12 & 0.00064 \\
\hline
\end{tabular}

required. The condition for the correct value of $\lambda_{0}$ is $N_{i}$ $=N_{i}^{*}\left(\lambda_{0} ; \lambda_{i}, f, \epsilon, d, a\right)$, where $N_{i}^{*}$ is the channel number calculated with Eq. 1 . When several spectral lines are available for the determination of $\lambda_{0}$, the best value of $\lambda_{0}$ should be determined by a least-squares fitting procedure.

For the experimental clata $\lambda_{i}, N_{i}$ in Tables I and II, approximate channel numbers were calculated: $N_{i}{ }^{*}=$ $N_{i}^{*}\left(\lambda_{i}\right)$ with Eq. 1 and the coefficients (20) and $N_{i}^{\prime}$ with Eq. 2 and the coefficients (21) and with the condition $\lambda_{i}{ }^{*}\left(N_{i}{ }^{\prime}\right)=\lambda_{i} \S$. The values of $N_{i}, N_{i}{ }^{*}$ and $N_{i}{ }^{\prime}$ in Tables I and II illustrate the following points:

1. Within the estimated accuracy of \pm 0.2 channel in the determination of the line positions $N_{i}$, the agreement between $N_{i}, N_{i}^{*}$ and $N_{i}^{\prime}$ is excellent. That means, with the given approximation $(k=1,2)$, Eqs. 1 and 2 are sufficiently accurate for the present data and are practically equivalent.

2. Statistical or systematic deviations from the ideal spatial linearity of the OMCD were not detectable by the measurement of known spectral lines. If there are such deviations, they are at most of the order $\lesssim 0.2 a=5$ $\mu \mathrm{m}$. This confirms the result obtained by Hamaguchi ${ }^{1}$ with an OMCD of the same type (PAR 1421). A small constant deviation of the specified value of $a$ cannot be detected by the present method, since only the ratio $a / f$ occurs in the coefficients of Eqs. 1 and 2, and $f$ has been treated as an adjustable parameter.

\section{LIMITATIONS OF THE FORMULAE}

From the excellent agreement between observed and calculated channel numbers in Tables I and II we conclude that, within the present range of application, systematic errors of $N^{*}$ and $N^{\prime}$ due to the approximations made are at most of the order of 0.2 channel. However, it is not obvious which of the two approximations ( 1 or 2 ) is the more accurate one. This can be easily found out by numerical calculation of the exact values $\mathrm{N}_{i}{ }^{e}\left(\lambda_{i}\right)$. In principle, an exact explicit expression for $\Delta N=(f / a)$ tan $\eta$ can be derived. For the numerical calculation of $\Delta N$,

$\S$ For the comparison of systematic errors in Eqs. 1 and 2, the channel numbers $N_{i}^{\prime}$ defined by $\lambda_{i}^{*}\left(N_{i}^{\prime}\right)=\lambda_{i}$ are more suitable than the approximate wavelengths $\lambda_{i}{ }^{*}=\lambda_{i}^{*}\left(N_{i}\right)$.
TABLE II. Literature wavelength values $\lambda_{i}$ of spectral lines and measured peak channel numbers $N_{i}$. The other channel numbers were calculated with the common parameters $N_{0}=500, \lambda_{0}=399.9088 \mathrm{~nm}$, and $\bar{\psi}_{0}=46.09329^{\circ}$ (cf. legend to Table I).

\begin{tabular}{crrrrr}
\hline$\lambda_{i} / \mathrm{nm}$ & \multicolumn{1}{c}{$N_{i}$} & \multicolumn{1}{c}{$N^{*}$} & \multicolumn{1}{c}{$N^{e}$} & \multicolumn{1}{c}{$N^{\prime}$} & $\left(\lambda^{*}-\lambda_{i}\right) / \mathrm{nm}$ \\
\hline 398.17711 & 31.0 & 31.27 & 30.99 & 31.03 & 0.00011 \\
398.39561 & 89.3 & 89.56 & 89.37 & 89.40 & 0.00041 \\
399.73919 & 453.7 & 453.41 & $\mathbf{4 5 3 . 4 1}$ & $\mathbf{4 5 3 . 4 1}$ & -0.00111 \\
399.80527 & 472.0 & $\mathbf{4 7 1 . 5 4}$ & 471.54 & 471.54 & -0.00163 \\
400.52414 & 670.5 & 670.29 & 670.30 & 670.30 & -0.00076 \\
400.97126 & 795.0 & 795.24 & 795.31 & 795.30 & 0.00106 \\
401.45308 & 931.0 & 931.06 & 931.27 & 931.24 & 0.00088 \\
\hline
\end{tabular}

however, no explicit expression for $\Delta N$ is needed. From Eqs. 5-8, 15, and 19 follows:

$$
\begin{aligned}
\psi_{0} & =\arcsin \left(\frac{1}{2} \Lambda_{0} / \cos \epsilon\right), \\
\xi & =\arcsin \left(\Lambda-\sin \alpha_{0}\right)-\beta_{0}, \\
\eta & =\arcsin \left[\Lambda-\sin \left(\alpha_{0}-\xi\right)\right]-\beta_{0}, \\
N & =N_{0}+(f / a) \tan \eta .
\end{aligned}
$$

With Eqs. 22-25, exact values $N_{\mathrm{i}}^{e}$ were calculated for all wavelengths $\lambda_{i}$ in Tables I and II. The comparison of $N_{i}{ }^{e}$ with $N_{i}^{*}$ and $N_{i}^{\prime}$ reveals that the agreement between $N_{i}{ }^{e}$ and $N_{i}{ }^{\prime}$ is in Table I considerably better and in Table II much better than that between $N_{i}{ }^{e}$ and $N_{i}{ }^{*}$.

In the case of an SGM, the superiority of Eq. 2 relative to Eq. 1 is obvious. The comparison of Eqs. 12c and 14c shows that-with increasing $\psi_{0}$-the cubic term in Eq. 1 increases and that in Eq. 2 decreases.

The superiority of approximation 2 in the case of a DGM is demonstrated more clearly in Table III. With respect to the data in Tables I and II, the main difference in Table III is a greater angle $\bar{\epsilon}\left(10^{\circ}\right)$, which is more typical for an in-plane Czerny-Turner or Ebert monochromator than is the very small value of $\bar{\epsilon}$ of our monochromator. For different values of $\lambda_{0}$ and $N^{\prime}=0,250,750$, and 1000,

TABLE III. Comparison of approximations 1 and 2 with $k \leq 2$ for a DGM with $d=\left(10^{6} / 3600\right) \mathrm{nm}, m=1, \bar{\epsilon}=10^{\circ}, f=600 \mathrm{~mm}$, and an OMCD with $a=0.025 \mathrm{~mm}$. With "approximate" values $N=0,250$, 750 , and 1000 , exact values $\lambda=\lambda^{*}\left(N^{\prime}\right)$ were obtained. With the values of $\lambda$, exact values $N^{*}$ were calculated with Eqs. 22-25 and approximate values $N^{\star}$ were calculated with Eq. 1 .

\begin{tabular}{lrrrr}
\hline & $\left(\lambda-\lambda_{0}\right) / \mathrm{nm}$ & \multicolumn{1}{c}{$N^{e}$} & $N^{\prime}-N^{e}$ & $N^{*}-N^{e}$ \\
\hline $\bar{\lambda}_{0}=100.00000 \mathrm{~nm}$ & -2.63374 & -0.105 & +0.105 & +0.130 \\
$\bar{\psi}_{0}=10.531542^{\circ}$ & -1.31366 & 249.987 & +0.013 & +0.016 \\
& 1.30724 & 750.013 & -0.013 & -0.016 \\
& 2.60806 & 1000.106 & -0.106 & -0.130 \\
$\lambda_{0}=250.00000 \mathrm{~nm}$ & -2.11865 & -0.089 & +0.089 & +0.203 \\
$\bar{\psi}_{0}=27.189954^{\circ}$ & -1.05374 & 249.989 & +0.011 & +0.025 \\
& 1.04256 & 750.011 & -0.011 & -0.025 \\
& 2.07394 & 1000.089 & -0.089 & -0.202 \\
$\lambda_{0}=400.00000 \mathrm{~nm}$ & -1.30673 & -0.023 & +0.023 & +0.503 \\
$\bar{\psi}_{0}=46.979292^{\circ}$ & -0.64640 & 249.997 & +0.003 & +0.062 \\
& 0.63247 & 750.002 & -0.002 & -0.061 \\
& 1.25102 & 1000.018 & -0.018 & -0.487 \\
$\lambda_{0}=417.00000 \mathrm{~nm}$ & -1.18624 & -0.004 & +0.004 & +0.593 \\
$\bar{\psi}_{0}=49.334181^{\circ}$ & -0.58614 & 250.000 & +0.000 & +0.073 \\
& 0.57217 & 750.000 & +0.000 & -0.072 \\
& 1.13037 & 999.997 & +0.003 & -0.572 \\
$\lambda_{0}=473.81585 \mathrm{~nm}$ & -0.71354 & 0.138 & -0.138 & +1.328 \\
$\bar{\psi}_{0}=60.000000^{\circ}$ & -0.35022 & 250.018 & -0.018 & +0.163 \\
& 0.33719 & 749.980 & +0.020 & -0.158 \\
& 0.66134 & 999.833 & +0.167 & -1.243 \\
\hline
\end{tabular}


the following quantities were calculated: $\lambda=\lambda\left(N^{\prime}\right)$ with Eqs. 2 and 21; $\Delta \lambda=\lambda-\lambda_{0}, N^{*}=N^{*}(\lambda)$ with Eqs. 1 and 20; and $N^{e}=N^{e}(\lambda)$ with Eqs. 22 to 25 . In Table III the following quantities are listed: $\lambda_{0}, \bar{\psi}_{0}, \lambda-\lambda_{0}, N^{e}, N^{\prime}-$ $N^{e}$, and $N^{*}-N^{e}$. The following observations can be made:

1. At small angles $\bar{\psi}_{0}\left(\bar{\psi}_{0} \leqslant 10^{\circ}, \lambda_{0} \leqslant 100 \mathrm{~nm}\right)$ approximations 1 and 2 are nearly equally accurate. Within the given range of $N$, the maximum systematic error of $N$ is $\approx 0.1$ channel.

2. The systematic errors are roughly proportional to ( $N$ $\left.-N_{0}\right)^{3}$, where $N_{0}=500$. This is expected, since the dominant contribution to the systematic errors must result from the omission of the cubic terms in Eqs. 1 and 2.

3. The systematic error of $N^{*}$ monotonously increases with increasing $\lambda_{0}$.

4. The systematic error of $N^{\prime}$ decreases with increasing $\lambda_{0}$, nearly vanishes at $\lambda_{0} \approx 417 \mathrm{~nm}\left(\bar{\psi}_{0} \approx 49^{\circ}\right)$, and then again increases with opposite sign. Obviously $B_{3}{ }^{d}$ changes sign at $\bar{\psi}_{0} \approx 49^{\circ}$. The very small residual systematic error of $N^{\prime}$ at this wavelength is mainly due to the omission of the $(\Delta N)^{4}$ term.

5. The grating-rotation range of monochromators is usually $0^{\circ} \leq \bar{\psi}_{0} \leq 60^{\circ}$. At $\bar{\psi}_{0}=60^{\circ}$, the systematic error of $N^{\prime}$ is still only of the order of 0.1 channel. That means, for the present range of $\eta(|\eta| \approx a|\Delta N| / f \leqslant$ $0.021 \mathrm{rad}$ or $\left.|\bar{\eta}| \leqslant 1.2^{\circ}\right)$, Eq. 2 with $k=1,2$ is accurate enough for all practical purposes. For the same range of $\bar{\psi}_{0}$ and the same average accuracy of $N^{*}$, Eq. 1 can be used in the range $|\eta| \leqslant 0.010 \mathrm{rad}$ or $|\bar{\eta}| \leqslant 0.6^{\circ}$.

Up to this point we have assumed the use of an ideal OMCD, and this assumption has been justified experimentally for the particular OMCD used in the present investigation. With other types of OMCD this ideal behavior is not observed, and Hamaguchi ${ }^{1}$ has proposed to use in this case virtual channel numbers instead of the measured channel numbers. Let $\bar{N}$ be a measured channel number and $N$ be, as before, the corresponding channel number measured with an ideal OMCD. If the relation $N=F(\bar{N})$ is known, all formulae of this paper can be applied to the calculated "virtual" channel numbers $N$.

Hamaguchi ${ }^{1}$ has used the term "virtual channel" with a different meaning. He assumed that the linear dispersion of an SGM was constant within the spectral range of an OMCD. For small rotational angles $\bar{\psi}_{0}$ of the grating, this assumption may be justified, but for the example given in Ref. 1 this assumption is already doubtful, as we will show now. With $f=600 \mathrm{~mm}, d=\left(10^{6} / 1200\right) \mathrm{nm}$, $\bar{\epsilon} \approx 10^{\circ}$ (our estimate), and $\lambda_{0}=515 \mathrm{~nm}$, we obtain: $\bar{\psi}_{0}=$ $18.286^{\circ}, A_{1}{ }^{s}=32.705 \mathrm{~nm}^{-1}$, and $A_{2}{ }^{s}=0.01199 \mathrm{~nm}^{-2}$. The spectral range of an OMCD with 1024 channels and $a=$ $0.025 \mathrm{~mm}$ is, in this case, $\approx 31 \mathrm{~nm}$. For $\lambda-\lambda_{0}=15.29$ nm, we obtain $A_{1}{ }^{s}\left(\lambda-\lambda_{0}\right)=500$ and $A_{2}^{s}\left(\lambda-\lambda_{0}\right)^{2}=$ 2.8. This result means that the neglect of the quadratic term in Eq. 1 must lead to systematic errors of $N$ up to the order of 1 channel, even if optimized values of $A_{1}^{s}$ and $\lambda_{0}$ are used. Hamaguchi gives an example for the justification of the neglect of a quadratic term in the case of an ideal OMCD, but despite much effort we have not been able to understand the relation between measured wavelengths (eq. 2 of Ref. 1) and the parameter values in table 1 of Ref. 1.

In conclusion, we believe that the procedure used by Hamaguchi ${ }^{1}$ has three disadvantages. First, the assumption of a constant linear dispersion within the whole spectral range of an OMCD can be justified only for small grating-rotation angles (e.g., $\left.\bar{\psi}_{0} \leqslant 10^{\circ}\right)$. For moderate or large values of $\bar{\psi}_{0}$, which are of interest in high-resolution spectroscopy, deviations from a constant linear dispersion are misinterpreted as distortions of a spectrum by the OMCD. Second, for a new center wavelength $\lambda_{0}$ a completely new and completely empirical determination of the coefficients $A_{k}{ }^{j}\left(\lambda_{0}\right)$ is required. Third, since in the completely empirical approach the $A_{k}{ }^{j}\left(\lambda_{0}\right)$ are no longer coefficients of a series expansion but coefficients of an arbitrary polynomial, many spectral lines are needed for a calibration, and the polynomial cannot be used for extrapolation beyond the range $\left(N_{\min }, N_{\max }\right)$ of the calibration.

\section{CONCLUSIONS}

For the combination of an ideal OMCD with a grating monochromator, the relation between channel number $N$ and wavelength $\lambda$ can be calculated from the channel spacing $a$ of the OMCD and the following physical parameters of the monochromator: grating period $d$, diffraction order $m$, Ebert angle $2 \epsilon$, focal length $f$, and center wavelength $\lambda_{0}$.

The exact functions $N=N(\lambda)$ and $\lambda=\lambda(N)$ can be approximated by truncated series expansions $N^{*}=N^{*}(\lambda)$ and $\lambda^{*}=\lambda^{*}(N)$ with terms up to the order $\left(\lambda-\lambda_{0}\right)^{n}$ or $\left(N-N_{0}\right)^{n}$.

With $n=2, \lambda \approx \lambda^{*}(N)$ is a better approximation than $N \approx N^{*}(\lambda)$. For a DGM in combination with an ideal OMCD, $\lambda \approx \lambda^{*}(N)$ with $n=2$ is sufficiently exact for all practical purposes, if $a\left|N-N_{0}\right| / f \leqslant 0.021$.

For an SGM, $\lambda \approx \lambda^{*}(N)$ or $N \approx N^{*}(\lambda)$ with $n=3$ are sufficiently exact for all practical purposes.

With proximity-focused MCP-intensified OMCDs, deviations from an ideal OMCD are typically less than 1 channel, in agreement with the specification given by manufacturers.

If the relation $N=F(\bar{N})$ between the channel numbers $N$ of an ideal OMCD and $\bar{N}$ of a real OMCD is known, "virtual" channel numbers $N$ can be calculated, to which the formulae of this paper apply.

\section{ACKNOWLEDGMENT}

This work was supported by the Deutsche Forschungsgemeinschaft (Sonderforschungsbereich 93, Photochemie mit Lasern).

1. H. Hamaguchi, Appl. Spectrosc. Rev. 24, 137 (1988).

2. G. W. Stroke, Diffraction Gratings, Encyclopedia of Physics, Vol. 29, Optical Instruments, S. Flügge, Ed. (Springer Verlag, Berlin, 1967), Sect. 18 , pp. $454-459$.

3. J. F. James and R. S. Sternberg, The Design of Optical Spectrometers (Chapman and Hall, London, 1969).

4. M. C. Hutley, Diffraction Gratings (Academic Press, London, 1982).

5. H. M. Crosswhite, The Iron-Neon Hollow-Cathode Spectrum, J. Res. Nat. Bur. Stand. 79a, 17 (1975). 